\title{
Fatores de riscos associados à ocorrência de helmintos gastrintestinais em cabras leiteiras mantidas em sistema de pastejo
}

\author{
Risk factors associated with the occurrence of gastrointestinal \\ helminths in dairy goats kept in grazing system
}

\author{
Jenevaldo Barbosa da Silva ${ }^{1 *}$; Adivaldo Henrique Fonseca ${ }^{2}$
}

\begin{abstract}
Resumo
Infecção por nematóides gastrointestinais pode interagir negativamente com a performace produtiva de cabras de leite, e em alguns casos induzir a mortalidade em rebanhos. Objetivou-se conhecer a influência da estação do ano, ordem de parto, periparto, lactação e padrão racial sobre a população de helmintos em cabras leiteiras. Foram utilizadas 31 cabras, sendo 15 Saanen e 16 F1 (1/2 Saanen + 1/2 Bôer). Destas, 13 eram de $1^{\circ}$ lactação e 18 de $2^{\circ}$ ou mais lactações. As maiores contagens de OPG ocorreram no verão e primavera, sendo o OPG dos animais Saanen superior $(\mathrm{p}<0,01)$ ao dos F1 ( $1 \frac{2}{2}$ Saanen $+1 / 2$ Bôer). Os animais de $1^{\circ}$ lactação apresentaram OPG superior $(\mathrm{p}<0,004)$ ao dos animais de $2^{\circ}$ ou mais lactação. Durante o periparto as maiores contagem de OPG para os dois padrões raciais foram na semana do parto $(\mathrm{p}<0,05)$, seguida pelo pós-parto e pré-parto. No periparto as cabras Saanen apresentaram OPG superior $(\mathrm{p}<0,02)$ aos F1 e as primíparas $(\mathrm{p}<0,008)$ em relação as pluríparas. Animais F1 são resistentes a helmintos quando comparado a animais Saanen, demonstrando que o cruzamento de raças resistentes com raças de alta produção é uma alternativa viável para aumentar a produtividade sem comprometer a estabilidade parasitaria do rebanho. O periparto constitui fator de risco associado á ocorrência de helmintos gastrintestinais, especialmente em cabras Saanen de primeira lactação.

Palavras-chave: Helmintos, lactação, periparto, resistência racial, Saanen, sazonalidade,
\end{abstract}

\begin{abstract}
Infection due to the gastrointestinal nematodes can negatively interact with the productive performance of dairy goats, and in some cases can induce mortality in the flocks. The objective was to study the influence of season, parturition order, postpartum, lactation and breed standard on the population of helminths in dairy goats. 31 goats were used, with 15 Saanen and 16 F1 (1/2 Boer $+1 / 2$ Saanen). Of these, 13 were from the 1 st lactation and 18 from the 2 nd or more lactations. The highest fecal egg counts occurred during the summer and spring, with FEC in Saanen higher $(\mathrm{p}<0.01)$ than in F1 animals $(1 / 2$ Boer $+1 / 2$ Saanen). The animals with 1st lactation showed higher FEC $(p<0.004)$ than the animals in 2nd or more lactation. During the peripartum the largest FEC for both breeds standards were in the week of the birth $(\mathrm{p}<0.05)$, followed by post-partum and pre-partum. In peripartum the Saanen goats showed higher FEC $(\mathrm{p}<0.02)$ than the F1 and primiparous $(\mathrm{p}<0.008)$ in relation to pluriparous. F1 animals are resistant to helminthes when compared to Saanen animals, demonstrating that crossing of resistant breeds with high production breeds is a viable alternative to increase productivity without compromising the parasitary stability of the herd. The peripartum period is a risk factor associated to the occurrence of gastrointestinal helminths, especially in Saanen goats from first lactation.

Key words: Helminths, postpartum, lactation, Saanen, seasonality and endurance breed
\end{abstract}

\footnotetext{
${ }^{1}$ Doutorando na Universidade Estadual Paulista, UNESP, Jaboticabal, SP. E-mail: jenevaldo@hotmail.com

${ }^{2}$ Prof. da Universidade Federal Rural do Rio de Janeiro, UFRRJ. Seropédica, RJ. E-mail: adivaldo@ufrrj.br

* Autor para correspondência
} 


\section{Introdução}

As infecções causadas por helmintos gastrintestinais são as doenças com maior impacto sobre a saúde de pequenos ruminantes (PERRY et al., 2002), podendo interagir negativamente com o desempenho produtivo de cabras leiteiras, e em alguns casos, induzir a mortalidade nos rebanhos (HOSTE et al., 2005). Além disso, o parasitismo por nematóides pode provocar alterações nas características nutricionais do leite, com uma diminuição no conteúdo de gordura (29,9\%), proteínas $(23,3 \%)$ e lactose $(19,6 \%)$ (RINALDI; VENEZIANO; CRINGOLI, 2007).

Caprinos são mais suscetíveis do que ovinos a verminoses gastrintestinais, apresentando maior produção de ovos nas fezes e maior parasitemia (ATTILI et al., 2004). Tratamentos anti-helmínticos são menos eficazes em caprinos e podem contribuir para a resistência de nematóides (MANFREDI et al., 2010). Estes resultados são particularmente importantes durante os programas de controle de infecções parasitárias, porque as cabras são muitas vezes consideradas similares aos ovinos e, tratadas com doses inferiores ao necessário (JACKSON; COOP, 2000; CABARET, 2000). Isso faz com que as medidas de controle tornem-se ineficazes contra os parasitas, e os caprinos podem ser responsabilizados pela manutenção e disseminação de populações de nematódeos resistentes em pequenos ruminantes (TORINA et al., 2004).

Os efeitos da infecção com nematóides dependem da idade do animal, raça, estado fisiológico, intensidade parasitária e das espécies envolvidas. A intensidade e prevalência do parasitismo pode variar com a estação do ano (SILVESTRE et al., 2000; GITHIGIA; THAMSBORG; LARSEN, 2001; PAPADOPOULOS et al., 2003), sendo que em caprinos a disponibilidade sazonal de larvas no pasto é característica fundamental na ocorrência e gravidade da doença. Vários fatores são conhecidos por afetar o nível de contaminação das pastagens e os padrões sazonais de larvas infectantes em uma determinada região geográfica. Estes incluem condições climáticas, práticas de manejo, estado nutricional e fisiológico dos animais (STEAR et al., 2000; GITHIGIA; THAMSBORG; LARSEN, 2001; HOSTE; LEVEQUE; DORCHIES, 2001).

O conhecimento epidemiológico relacionado ao ambiente, aos parasitos e a fatores fisiológicos intrínsecos aos caprinos é fundamental para a implantação de um sistema de controle estratégico que seja efetivo contra os helmintos gastrintestinais, uma vez que trabalhos relacionados ao tema, em sua grande maioria, foram feitos com a espécie ovina (HOUDIJK et al., 2000; MANDONNET et al., 2001; PINTO et al., 2008). Essa vermifugação, se necessária, seria utilizada no período mais adequado e auxiliaria na diminuição da carga parasitária e da contaminação do ambiente, no qual, na maioria das vezes, animais jovens, mais suscetíveis irão compartilhar.

O conhecimento dos fatores de risco para helmintoses é fundamental para o uso de antihelmínticos de forma orientada visando reduzir a propagação e desenvolvimento de resistência em populações de nematóides (VATTA et al., 2001; GARIPPA et al., 2008). Os resultados do atual estudo podem fornecer $r$ base racional para a utilização de tratamentos de forma seletiva em rebanhos de caprinos leiteiros. Tais tratamentos seriam de grande interesse para reduzir o custo de produção e a disseminação da resistência, cuja prevalência é particularmente alta nos caprinos (CABARET, 2000). O objetivo do estudo foi conhecer a influência dos fatores de risco associados á ocorrência de verminose: estação do ano, periparto, lactação e padrão racial sobre a contagem de ovos de helmintos gastrintestinais nas fezes de cabras leiteiras.

\section{Material e Métodos}

O estudo foi realizado no Setor de Caprinocultura, Instituto de Zootecnia da Universidade Federal Rural do Rio de Janeiro (UFRRJ), situada na microrregião Metropolitana da Cidade do Rio de 
Janeiro (latitude $22^{\circ} 45^{\prime} \mathrm{S}$, longitude $43^{\circ} 41^{\prime} \mathrm{W}$ e altitude de 33 metros). Segundo a classificação de Köppen (KÖPPEN; GEIGER, 1928) o clima da região pertence à classe Aw, caracterizado por inverno seco e verão chuvoso e quente, com precipitação anual de $1300 \mathrm{~mm}$, inverno pouco pronunciado; temperatura média anual de $24{ }^{\circ} \mathrm{C}$ e umidade relativa do ar de $70 \%$.

Para avaliar a distribuição estacional da população de helmintos gastrintestinais, foram selecionadas aleatoriamente 31 cabras leiteiras em um rebanho de 100 matrizes, obedecendo o modelo de amostragem estratificada proporcional. $\mathrm{Na}$ avaliação da influencia do padrão racial sobre a intensidade parasitaria, foram utilizadas 15 cabras Saanen e 16 F1 ( $1 / 2$ Saanen + $1 / 2$ Bôer). Por fim, foi avaliado o efeito da ordem de parto sobre a carga parasitaria, sendo utilizadas 13 fêmeas de $1^{\circ}$ lactação (primíparas) e 18 de $2^{\circ}$ ou mais lactações (pluríparas).

As cabras foram mantidas em sistema de pastejo continuo de Brachiaria humidicula, sob densidade de 20 animais/ha. Durante a estação seca do ano os animais foram alimentados com volumoso de capim elefante (Pennisetum purpureum Schum cv. Cameroom) picado no cocho diariamente, ração concentrada com $19 \%$ de proteína bruta e sal mineral ad libitum. O manejo reprodutivo empregado preconizava a utilização de monta natural, sendo as fêmeas cobertas entre fevereiro e março, e as parições concentradas nos meses de julho e agosto.

Um mês antes do inicio do experimento, todos os animais foram vermifugados. O anti-helmíntico utilizado continha o princípio ativo: Moxidectin (0.2 mg/kg, Cydectin ${ }^{\circledR}$, Fort Dodge, Brazil) sendo utilizada dose única. Durante o estudo foi utilizado vermifugação conforme contagem individual de OPG, quando cada vez que esse valor atingia 4000 o animal recebio o tratamento anti-helmíntico. Essa metodologia foi utilizada para evitar que os animais morressem ou inviabilizasse o sistema produtivo.
Foram realizadas semanalmente coletas de amostras fecais de todas as cabras entre o período de abril de 2008 a março de 2009. Para a contagem de ovos por grama de fezes (OPG) de foi realizada técnica McMaster de acordo a Gordon e Whithlock (1939). A recuperação das larvas foi feita através da coprocultura segundo Roberts e O’Sullivan (1950). A identificação das larvas de terceiro estágio pertencentes à superfamília Trichostrongiloidea recuperadas na coprocultura foi feita segundo a chave de Keith (1953). Utilizando o modelo proposto por Ueno e Gonçalves (1998) estimou-se o número de OPG, a carga parasitária e a equivalência patogênica de cada gênero de nematódeo gastrintestinal encontrado.

Os dados sobre as variáveis climáticas foram fornecidos pelo Instituto Nacional de Metereologia (INMET) - estação Ecologia Agrícola do Km 47, Convênio Pesagro-Rio. Para análise estatística os dados referentes a contagens de ovos por grama de fezes (OPG) foram transformados inicialmente em $\log 10$, afim de normaliza-los. Para avaliação quantitativa dos diferentes parâmetros avaliados, foram utilizadas regressão linear e teste $t$ de Student a $5 \%$ de significância. Os procedimentos operacionais foram realizados com o auxílio do software estatístico Epi Info $\AA$, version 3.5 (CENTER FOR DISEASE CONTROL, 2008).

\section{Resultados e Discussão}

Os resultados referentes á contagem de ovos de helmintos da superfamília Trichostrongyloidea (OPG) presentes nas fezes das cabras Saanen e F1 $(1 / 2$ Saanen $x 1 / 2$ Boer) durante as quatro estações do ano estão apresentados na Figura 1. Os maiores valores de OPG para os dois grupos foram no verão e primavera, embora não tenha sido encontrada diferença estatística $(p>0,05)$ entre as estações. O número de ovos nas fezes dos animais Saanen foi significativamente maior $(\mathrm{p}<0,01)$ do que nos animais F1. 
Figura 1. Variação estacional da contagem de ovos de helmintos da superfamília Trichostrongyloidea nas fezes de cabras Saanen e F1 (1/2 Saanen x 1/2 Boer), Instituto de Zootecnia da UFRRJ, 2008-2009.

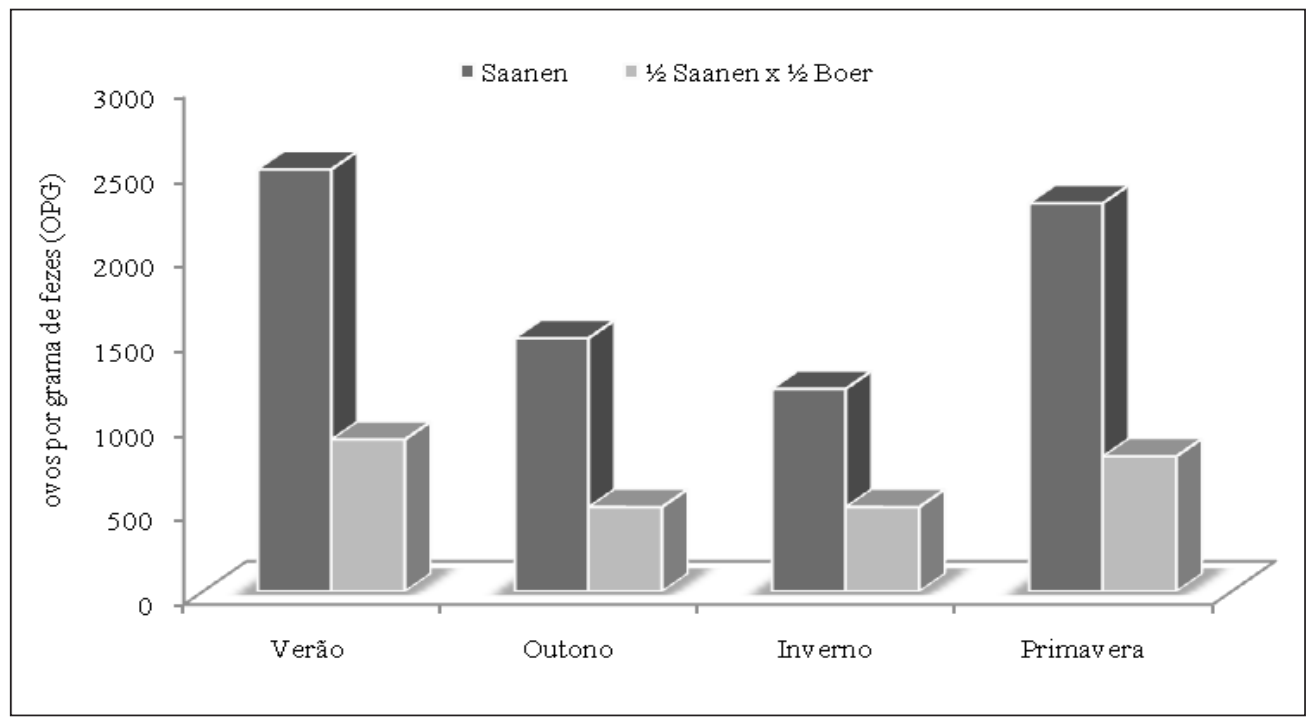

Fonte: Elaboração dos autores.

A variação sazonal da contagem de ovos nas fezes demonstrou que animais da raça Saanen são menos resistentes a helmintos gastrintestinais do que animais F1. Estes resultados corroboram estudos anteriormente realizados que observaram maior suscetibilidade à infecção Trichostrongyloidea em animais de raças puras de alto nível de produção leiteira (CHARTIER et al., 2000; HOSTE; LEVEQUE; DORCHIES, 2001, HOSTE et al., 2002b). Foi proposto que tal diferença de intensidade parasitaria poderia ser explicado pela demanda excessiva de nutrientes relacionados à alta produção de leite (COOP; KYRIAZAKIS, 1999).

Os resultados comparativos referentes à contagem de ovos nas fezes de cabras primíparas e pluríparas durante as quatro estações do ano estão expressos na Figura 2. Os animais de primeira lactação apresentaram contagem de ovos nas fezes significativamente superior $(p<0,004)$ ao dos animais de mais de uma lactação.

A maior susceptibilidade dos animais primíparos, deve-se provavelmente ao manejo e o uso inadequado de anti-helmíntico. Na maioria das fazendas, as fêmeas jovens, criadas para repor o plantel, são mantidas separadas dos demais animais, dificultando o contato prévio com helmintos e conseqüentemente o desenvolvimento de resistência. Em segundo lugar, a administração de tratamentos anti-helmínticos em caprinos baseiase normalmente em estimativa de peso médio (HOSTE et al., 2000), sendo que superestimação ocorre comumente para caprinos adultos enquanto que isto é menos provável em cabras de primeira lactação, cujo peso é geralmente mais leve (HOSTE et al., 2002b).

A contagem de ovos nas fezes durante o período periparto para os animais Saanen e F1 está representada na Figura 3. Quando confrontado os valores médios do OPG do periparto e das estações do ano, observou-se que o primeiro foi significativamente superior $(p<0,01)$. As maiores contagem de OPG nos dois grupos foram na semana do parto $(\mathrm{p}<0,05)$, seguida pelo pós-parto e pré-parto. Os animais Saanen apresentaram OPG superior $(\mathrm{p}<0,02)$ aos $\mathrm{F} 1$ durante as três fases do periparto. 
Figura 2. Variação estacional da contagem de ovos de helmintos da superfamília Trichostrongyloidea nas fezes de cabras primíparas e pluríparas, Instituto de Zootecnia da UFRRJ, 2008-2009.

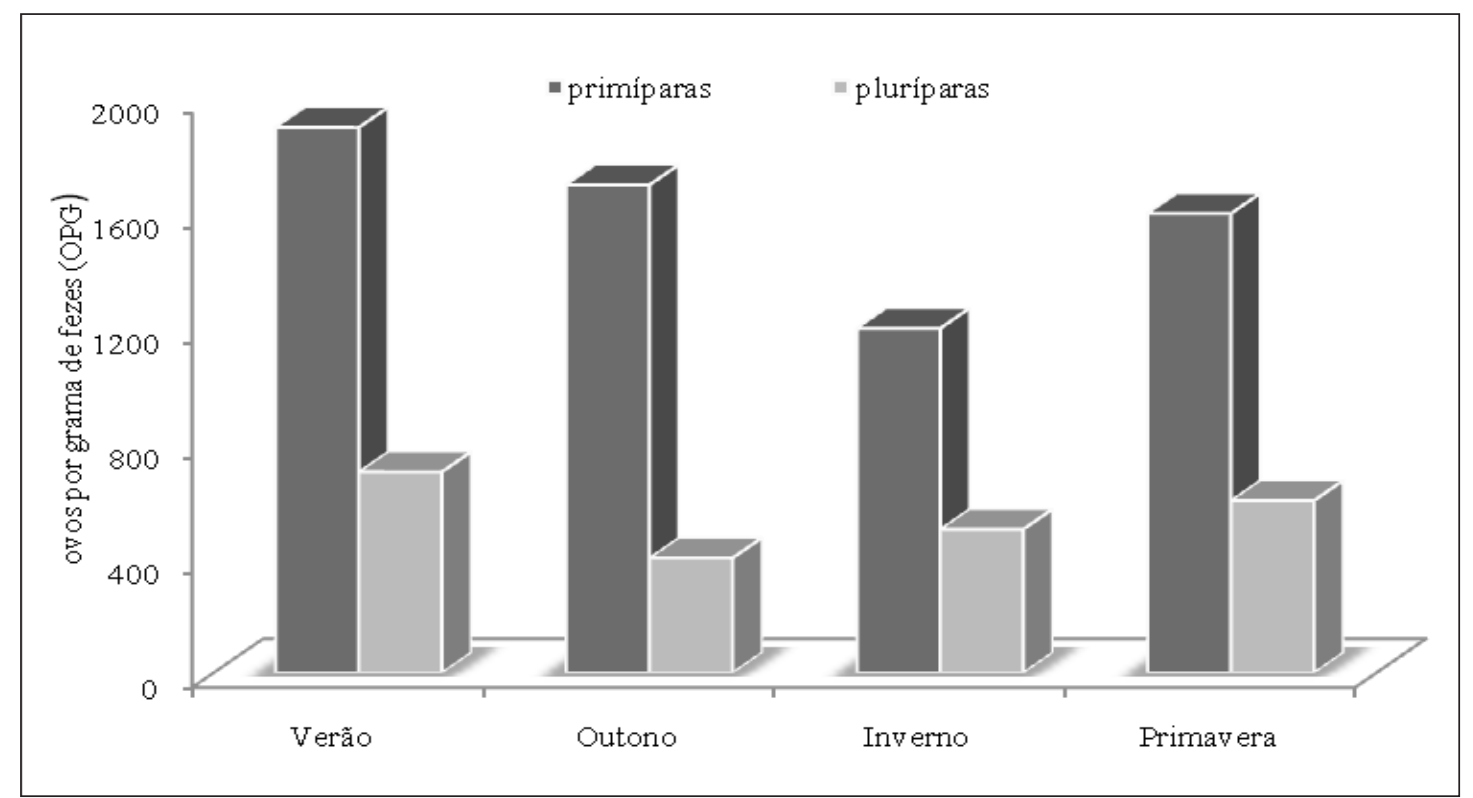

Fonte: Elaboração dos autores.

Figura 3. Variação da contagem de ovos de helmintos da superfamília Trichostrongyloidea nas fezes de cabras Saanen e F1 ( $1 / 2$ Saanen x 1/2 Bôer), durante o periparto, Instituto de Zootecnia da UFRRJ, 2008-2009.

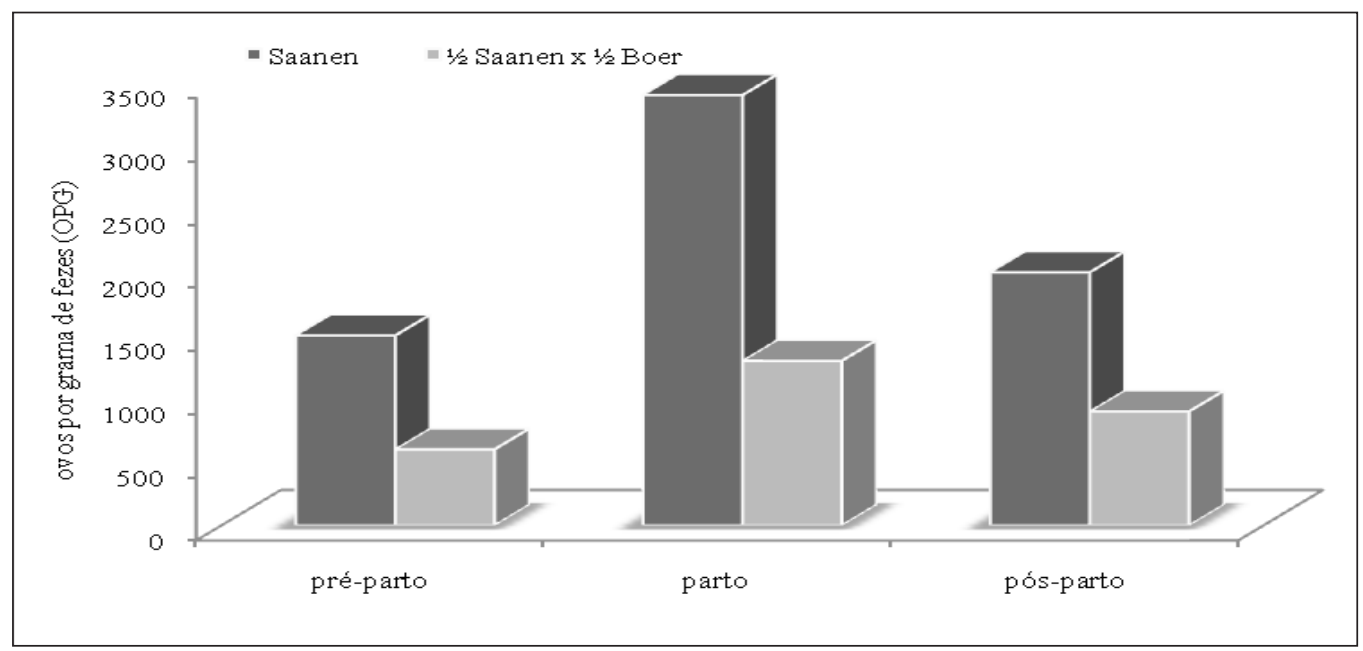

Fonte: Elaboração dos autores.

A variação do OPG em função do parto observada corrobora pesquisas anteriormente realizadas em caprinos leiteiros (PATTERSON et al., 1996; CHARTIER; HOSTE, 1998; SILVA et al., 2011). Em ovinos tem sido sugerido que o padrão de aumento de ovos devido ao periparto poderia ser em função da quantidade de leite secretado (JANSEN,
1973), mas esta hipótese ainda não foi explorada em cabras leiteiras.

Os resultados demonstram que cabras de primeira lactação apresentaram maior abundância de parasitárias gastrintestinais, comprovando que a ordem de parto constitui fator de risco para 
o desenvolvimento de infecções por helmintos. Estes resultados corroboram estudos anteriormente realizados, que sugerem a hipótese de que a maior receptividade a helmintos das cabras de primeira lactação é devida à falta ou à baixa intensidade de contatos anteriores com helmintos e a ausência de uma resposta imune capaz de regular as populações de vermes (VALLADE et al., 2000; HOSTE et al., 2002a).

Figura 4. Variação da contagem de ovos de helmintos da superfamília Trichostrongyloidea nas fezes de cabras primíparas e pluríparas, durante o periparto, Instituto de Zootecnia da UFRRJ, 2008-2009.

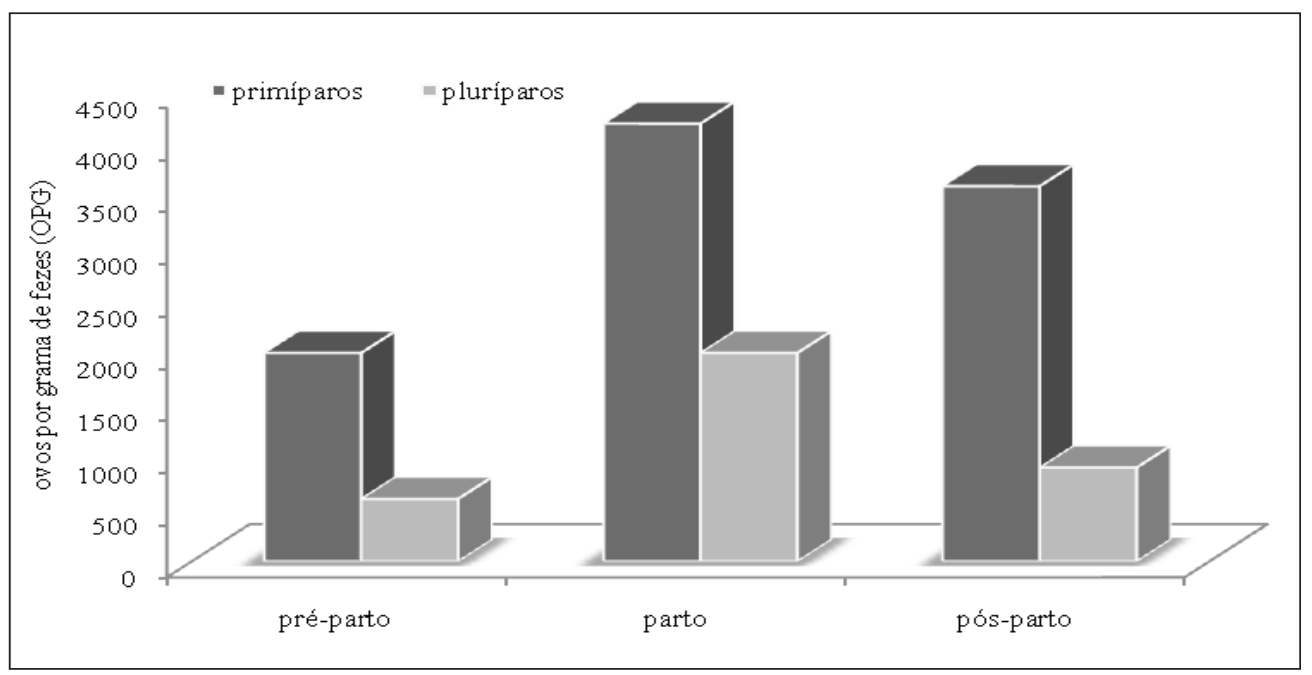

Fonte: Elaboração dos autores.

Em cabras leiteiras, as diferenças observadas na receptividade e suscetibilidade a helmintos dentro de um rebanho dependendo da idade ou do nível de produção foram usadas para fornecer a lógica para a utilização de aplicação seletiva de antihelmínticos (HOSTE et al., 2002a). Os resultados atuais confirmam que as cabras na primeira lactação devem representar o alvo da fiscalização específica e possível tratamento seletivo nesse grupo de animais sob maior risco do rebanho. Tais tratamentos seletivos seriam de grande interesse para diminuir a disseminação da resistência anti-helmíntica, cuja prevalência é particularmente alta nos caprinos (CABARET, 2000).

Hoste et al. (2002b) sugerem que um pequeno número de animais do rebanho caprino exibem um
Os resultados da contagem de OPG dos animais primíparos e pluríparos, durante o periparto estão na Figura 4. As maiores contagem de OPG nos dois grupos foram na semana do parto $(\mathrm{p}<0,05)$, seguida pelo pós-parto e pré-parto. Os animais primíparos apresentaram OPG superior $(\mathrm{p}<0,008)$ aos dos animais pluríparos durante as três fases do periparto. 
Figura 5. Variação sazonal da população de helmintos da superfamília Trichostrongyloidea em cabras leiteiras, Instituto de Zootecnia da UFRRJ, 2008-2009.

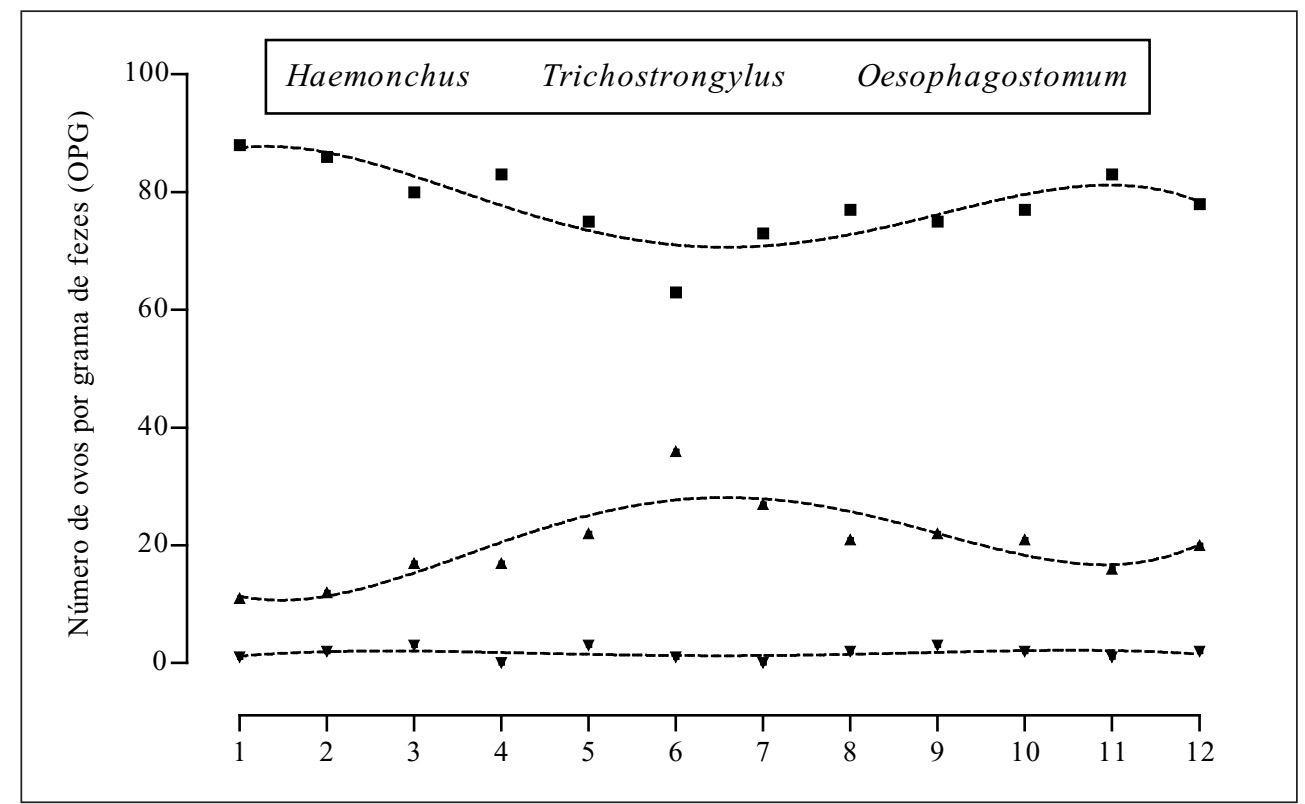

Fonte: Elaboração dos autores.

Os resultados corroboram Mahieu et al. (2007) e Silva et al. (2011) que observaram que os gêneros de maior frequência em área tropical são Haemonchus e Trichostrongylus. Pimentel Neto (1976) em estudos na região de baixada do Estado do Rio de Janeiro observou uma tendência de redução do número de larvas de Haemonchus durante a estação seca do ano, ao passo que o número de larvas de Trichostrongylus aumenta, denominando este fenômeno de inversão Haemonchus/Trichostrongylus.

Durante o período de temperaturas mais baixas do ano foi observada uma redução dos valores percentuais de Haemonchus. Este achado se deve ao fato do Trichostrongylus ocorre principalmente em regiões temperadas e subtropicais. Sendo esta última região favorável para a tolerância deste parasito às baixas temperaturas, principalmente, no outono e inverno (RAMOS et al., 2004). Além disso, em condições subtropicais não ocorrem neves intensas, este fator é limitante à sobrevivência das larvas no solo.

Deste modo, a verminose tem se mostrado endêmina na maioria dos rebanhos ovinos criados nos trópicos. A ineficiência dos principais grupos de bases antihelminticas em decorrência do advento da resistência dos nematóides, aliados a busca dos consumidores por produtos oriundos de sistemas de produção sustentáveis, tem levado os pesquisadores a buscarem novas saídas para o controle de helmintos. Neste senário, a adoção de medidas de tratamento seletivo mirando os animais são responsáveis pela maior contaminação das pastagens e a utilização de sistemas de pastejo rotativo parece ser a alternativa mais viável. Para isso, é de fundamental importância conhecer as classes de animais responsáveis mais severamente atingidas pelos helmintos gastrintestinais dentro de um rebanho.

Deste modo, o periparto constituiu um fator de risco associado á ocorrência de helmintos gastrintestinal, especialmente em cabras Saanen de primeira lactação. Os animais F1 ( $1 / 2$ Saanen x 1/2 Bôer) foram resistentes a helmintos gastrintestinais, demonstrando que o cruzamento de raças resistentes com raças de alta produção e uma alternativa viável para aumentar a produtividade sem comprometer a estabilidade parasitaria do rebanho. 
A utilização de tratamentos seletivos, tendo como alvo os animais responsáveis pela maior contaminação das pastagens, aliado ao pastejo rotacionado parece ser uma alternativa viável para a produção sustentável, evitando deste modo o aparecimento de resistência parasitária e morte de animais das classes mais sensíveis do plantel.

\section{Agradecimentos}

Ao CNPq e FAPERJ pelo apoio financeiro.

\section{Referências}

ATTILI, A. R.; AYALA, C.; TRALDI, G.; FURBETTA, R.; HABLUETZEL, A. Endo-parasitic infection patterns in angora goats and merino sheep in a fibre animal farm in Central Italy. Parassitologia, Košice, v. 46, p. 23, 2004. Suplemento 1.

CABARET, J. Anthelmintic resistance in goats: from fiction to facts. In: INTERNATIONAL CONFERENCE ON GOATS, 7., 2000, Tours. Proceedings... Tours: [s.n.], may, 2000. p. 793-794.

CENTER FOR DISEASE CONTROL. Epinfo. 2008. Disponível em: <http://www.cdc.gov/epiinfo $>$. Acesso em: 25 out. 2009.

CHARTIER, C.; ETTER, E.; HOSTE, H.; PORS, I.; MALLEREAU, M. P.; BROQUA, C.; MALLET, S.; KOCH, C.; MASSE, A. Effects of the initial level of milk production and of the dietary protein intake on the course of natural nematode infection in dairy goats. Veterinary Parasitology, Amsterdam, v. 92, n. 1-2, p. 1-13, 2000.

CHARTIER, C.; HOSTE, H. Repeated infections with Haemonchus contortus and Trichostrongylus colubriformis in dairy goats: comparison of resistant and susceptible animals. Parasitology Research, Berlin, v. 84, n. 3, p. 249-253, 1998.

COOP， R. L.; KYRIAZAKIS， I. Nutrition-parasite interaction. Veterinary Parasitology, Amsterdam, v. 84, n. 3-4, p. 187-204, 1999.

GARIPPA, G.; BUFANO, G.; CAROLI, A.; CARTA, A.; CRINGOLI, G.; DE NARDO, F.; FILIP-PINI, G.; LEORI, S. G.; MONIELLO, G.; RONCHI, B. Realtà e prospettive dell'allevamento dei piccoli ruminanti in Italia. Large Animal Research, Cambridge, v. 4, p. 40-43, 2008. Suplemento 1.
GITHIGIA, S. M.; THAMSBORG, S. M.; LARSEN, M. Effectiveness of grazing management in controlling gastrointestinal nematodes in weaner lambs on pasture in Denmark. Veterinary Parasitology, Amsterdam, v. 99, n. 1-2, p. 15-27, 2001.

GORDON, H. M. C. L.; WHITLOCK, H. V. A new technique for counting nematode eggs in sheep faeces. Journal of the Council for Scientific and Industrial Research, Melbourne, v. 12, n. 1, p. 50-52, 1939.

HOSTE, H.; CHARTIER, C.; ETTER, E.; GOUDEAU, C.; SOUBIRAC, F.; LEFRILEUX, Y. A. questionnaire survey on the practices adopted to control gastrointestinal nematode parasitism in dairy goat farms in France. Veterinary Research Communications, Edinburgh, v. 24, n. 7, p. 459-469, 2000.

HOSTE, H.; CHARTIER, C.; LE FRILEUX, Y. Control of gastrointestinal parasitism with nematodes in dairy goats by treating the host category at risk. Veterinary Research, Les Ulis, v. 33, n. 5, p. 531-546, 2002a.

HOSTE, H.; LE FRILEUX, Y.; GOUDEAU, C.; CHARTIER, C.; PORS, I.; BROQUA, C.; BERGEAUD, J. P. Distribution and repeatibility of nematode faecal egg counts in dairy goats: a farm survey and implication for worm control. Research Veterinary Science, Oxford, v. 72, n. 1, p. 211-215, 2002 b.

HOSTE, H.; LEVEQUE, H.; DORCHIES, P. H. Comparison of nematode infections of the gastrointestinal tract in Angora and dairy goats in a rangeland environment: relations with the feeding behaviour. Veterinary Parasitology, Amsterdam, v. 101, n. 1-2, p. 127-135, 2001.

HOSTE, H.; TORRES-ACOSTA, J. F.; PAOLINI, V.; AGUILAR-CABALLERO, A.; ETTER, E.; LEFRILEUX, Y.; CHARTIER, C.; BROQUA, C. Interactions between nutrition and gastrointestinal infections with parasitic nematodes in goats. Small Ruminant Research, Thessaloniki, v. 60, n. 1-2, p. 141151, 2005.

HOUDIJK, J. G. M.; KYRIAZAKIS, I.; JACKSON, F.; HUNTLEY, J. F.; COOP, R. L. Can an increased metabolizable protein intake affect the periparturient relaxation in immunity against Teladorsagia circumcincta in sheep? Veterinary Parasitology, Amsterdam, v. 91, n. 1-2, p. 43-62, 2000.

JACKSON, F.; COOP, R. L. The development of anthelmintic resistance in sheep nematodes. Parasitology, Cambridge, v. 120, p. 95-107, 2000.

JANSEN, J. The spring rise phenomenon in sheep. II. The influence of lactation. Helminthologia, Košice, v. 14, n. 1, p. 261-270, 1973. 
KEITH, R. K. The diferentiation of the infective larvae of some commom nematode. Australian Journal of Zoology, New Jersey, v. 1, n. 2, p. 223-235, 1953.

KÖPPEN, W.; GEIGER, R. Klimate der Erde. Gotha: Verlag Justus Perthes. 1928. Wall-map $150 \mathrm{~cm} \times 200 \mathrm{~cm}$.

MAHIEU, M.; ARQUET, R.; KANDASSAMY, T.; MANDONNET, N.; HOSTE, H. Evaluatio of targeted drenching using Famacha(C method in Creole goat: Reduction of anthelminti use, and effects on kid production and pasture contamination. Veterinary Parasitololy, Amsterdam, v. 146, n. 2-3, p. 135-147, 2007.

MANDONNET, N.; AUMONT, G.; ARQUET, R.; VARO, H.; GRUNER, L.; BOUIX, J.; VU TIEN KHANG, J. Assessment of genetic variability of resistance to gastrointestinal nematode parasites in Creole goats in the humid tropics. Journal of Animal Science, Champaign, v. 79, n. 7, p. 1706-1712, 2001.

MANFREDI, M. T.; DI CERBOA, A. R.; ZANZANI, S.; STRADIOTTO, K. Breeding management in goat farms of Lombardy, northern Italy: Risk factors connected to gastrointestinal parasites. Small Ruminant Research, Thessaloniki, v. 88, n. 2, p. 113-118, 2010.

PAPADOPOULOS, E.; ARSENOS, G.; SOTIRAKI, S.; DELOGIANNIS, C.; LAINAS, T.; ZYGOYIANNIS, D. The epizootiology of gastrointestinal nematode parasites inGreek dairy breeds of sheep and goats. Small Ruminant Research, Thessaloniki, v. 47, n. 1, p. 193-202, 2003.

PATTERSON, D. M.; JACKSON, F.; HUNTLEY, J. F.; STEVENSON, L. M.; JONES, D. G.; JACKSON, E.; RUSSEL, A. J. F. Studies on caprine responsive ness to nematodiasis: segregation of male goats into responders and non-responders. International Journal for Parasitology, Valencia, v. 26, n. 11, p. 187-194, 1996.

PERRY, B. D.; MCDERMOTT, J. J.; RANDOLPH, T. F.; SONES, K. R.; THORNTON, P. K. Investing in animal health research to alleviate poverty. Nairobi, Kenya:International Livestock Research Institute, 2002.

PIMENTEL NETO, M. Epizootiologia da hemoncose em bezerros de gado de leite no Estado do Rio de Janeiro. Pesquisa Agropecuária Brasileira, Brasília, v. 11, n. 9, p. 101-114, 1976.

PINTO, J. M. S.; OLIVEIRA, M. A. L.; ÁLVARES, C. T.; COSTA-DiAS, R.; SANTOS, M. H. Relação entre o periparto e a eliminação de ovos de nematóides gastrintestinais em cabras anglo nubiana naturalmente infectadas em sistema semi-extensivo de produção. Revista Brasileira de Parasitologia Veterinária, Jaboticabal, v. 17, p. 138-143, 2008. Suplemento 1.
RAMOS, C. I.; BELLATO, V.; SOUZA, A. P.; AVILA, V. S.; COUTINHO, G. C.; DAlAGNOL, C. A. Epidemiologia das helmintoses gastrintestinais de ovinos no planalto catarinense. Ciência Rural, Santa Maria, v. 34, n. 6, p. 1889-1895, 2004.

RINALDI, L.; VENEZIANO, V.; CRINGOLI, G. Dairy goat production and the importance of gastrointestinal strongyle parasitism. Transactions of the Royal Society of Tropical Medicine and Hygiene, London, v. 103, n. 12, p. 745-746, 2007.

ROBERTS, F. H. S.; O'SULLIVAN, P. J. Methods for eggs counts and larval cultures for strongyles infecting the gastro-intestinal tract of cattle. Australian Journal of Agriculture Research, v. 1, n. 1, p. 99-192, 1950.

SILVA, J. B.; FAGUNDES, G. M.; FONSECA, A. H. Dynamics of gastrointestinal parasitoses in goats kept in organic and conventional production systems in Brazil. Small Ruminant Research, Thessaloniki, v. 98, n. 1-3, p. 35-38, 2011.

SILVESTRE, A.; CHARTIER, C.; SAUVE, C.; CABARET, J. Relation- ship between helminth species diversity, intensity of infection and breeding management in dairy goats. Veterinary Parasitology, Amsterdam, v. 94, n. 1-2, p. 91-105, 2000.

STEAR, M. J.; MITCHELL, S.; STRAIN, S.; BISHOP, S. C.; MCKELLAR, Q. A. The influence of age on the variation among sheep in susceptibility to natural nematode infection. Veterinary Parasitology, Amsterdam, v. 89, n. 1, p. 31-36, 2000.

TORINA, A.; DARA, S.; MARINO, A. M. F.; SPARAGANO, O. A. E.; VITALE, F.; REALE, S.; CARACAPPA, S. Study of gastrointestinal nematodes in Sicilian sheep and goats. Annals of the New York Academy of Sciences, New York, v. 1026, n. 54, p. 187194, 2004.

UENO, H.; GONÇALVES, P. C. Manual para diagnóstico das helmintoses $d e$ ruminantes. $4^{\text {th }}$ ed. Tokyo: Japan International Cooperation Agency, 1998. 143 p.

VALLADE, S.; HOSTE, H.; GOUDEAU, C.; BROQUA, C.; LAZARD, K.; LEFRILEUX, Y.; CHARTIER, C.; ETTER, E. Relationship between nematode parasitism of the digestive tract and the characteristics of dairy goat farms in two French regions. Revue de Médecine Vétérinaire, Toulouse, v. 12, n. 151, p. 1131-1138, 2000.

VATTA, A. F.; LETTY, B. A.; VAN DER LINDE, M. J.; VAN WIJK, E. F.; HANSEN, J. W.; KRECEK, R. C. Testing for clinical anaemia caused by Haemonchus spp. In goats farmed under resource-poor conditions in South Africa using an eye colour chart developed for sheep. Veterinary Parasitology, Amsterdam, v. 99, n. 1, p. 1-14, 2001. 
